Original Article

\section{Comparison of single-stage and two-stage ERCP Laparoscopic Cholecystectomy}

\author{
Fatima Zehra Khan ${ }^{1}$, Mir Arsalan Ali ${ }^{1}$, Shafiq Ur Rehman', \\ Syed Mustafa Ali Shah ${ }^{2}$, Maheen Nisar $^{2} \&$ \\ Zohaib Jawed Abubaker ${ }^{2}$ \\ ${ }^{1}$ Dr. Ziauddin Hospital, Karachi-Pakistan \\ ${ }^{2}$ Department of General Surgery, Ziauddin University, Karachi-Pakistan
}

DOI:10.29052/IJEHSR.v8.i2.2020.75-80

Corresponding Author Email:

drfatima.khan@gmail.com

Received 21/01/2020

Accepted 11/04/2020

Published 01/06/2020

\section{(c) (1)}

(C) The Author(s). 2020 Open Access This article is distributed under the terms of the Creative Commons Attribution 4.0 International License (http://creativecommons.org/licenses/by/4.0/)

OPEN ACCESS

\title{
Abstract
}

Background: Gallbladder surgeries are among the commonest major abdominal operations and to continuously improve the procedure many variants have developed that need evidence-based comparison in terms of frequency common bile duct (CBD) clearance, the mean operative time and hospital stay, and rate of adverse outcomes (post-operative pancreatitis). Through this study, we aimed to compare the surgical outcome of single-stage Endoscopic Retrograde Cholangio-Pancreatography (ERCP) with LC in comparison to two-stage ERCP.

Methodology: This prospective study was conducted on a sample of 190 diagnosed patients (chosen via nonprobability consecutive sampling) of cholelithiasis with choledocholithiasis, aged between 20 to 70 years who were scheduled to undergo surgery at the Ziauddin University Hospital, Karachi. The patients were divided into two equal groups (Group A: Single-stage ERCP \& LC, and Group B: Two-stage ERCP followed by interval LC). After taking written informed consent, data pertaining to surgical outcome were recorded onto a pre-structured questionnaire and analyzed using SPSS Version 23.0.

Results: The demographic and clinical characteristics of both the groups were similar and so were the success rates. Adverse events free and positive outcomes were reported in $95.79 \%$ and $90.53 \%$ of the patients in groups A and B respectively. Post-operative pancreatitis was reported more often among the patients of group B. The length of hospital stay and costs were lower among Group A patients despite the longer operative times in this group.

Conclusion: Patients in group A yield better surgical outcomes, giving single-stage ERCP \& LC the edge over two-stage ERCP followed by interval LC. Thus, single-stage ERCP \& LC is recommended as the method of choice.

\section{Keywords}

Cholelithiasis, Choledocholithiasis, Laparoscopic Cholecystectomy (LC), Endoscopic Retrograde cholangiopancreatography (ERCP), Surgical Outcome.

\section{Check for updates}




\section{Introduction}

Cholelithiasis, Choledocholithiasis disease is the cause behind $16 \%$ of all reported abdominal pain complaints and accounts for up to $21 \%$ of all patients presenting to the hospital with a surgical abdomen, most the times, the ultimate cure is cholecystectomy ${ }^{\mathrm{I}}$. Initially, cholecystectomy was performed by major abdominal incision, however, with the introduction of Laparoscopic Cholecystectomy in 1987, this is the preferred procedure however in some cases the surgeons are required to do the open Cholecystectomy. Laparoscopic Cholecystectomy has its advantages due to its minimal level of invasion, less trauma inflicted during surgery, decreased risk of bleeding and infection, smaller surgical scar and shortened length of stay at the hospital as compared to the open alternative $e^{2}$.

The prevalence of cholelithiasis in the Pakistani population is around $10.4 \%{ }^{3}$, furthermore, $18 \%$ of the patients with cholelithiasis have associated choledocholithiasis with the incidence peaking in the Pakistani population at the age of 30 to 44 years. It has been reported by Samra et al., that out of 400 diseased gallbladder patients $320(80 \%)$ had gallstones. ERCP has become the gold standard for isolated common bile duct stones ${ }^{4}$. The commonly employed strategy is thus ERCP with sphincterotomy followed by extraction of common bile duct stone via Dormia basket or balloon which in turn followed by LC. The procedure can be performed in a single-stage or two stages. The other management options for CBD stones include laparoscopic exploration, open exploration, extracorporeal shock wave lithotripsy and laser lithotripsy to name a few ${ }^{5}$.

Single-stage ERCP with LC in a single sitting is associated with higher success rates of CBD clearance and reduced hospital stay and operation time. However, the technique requires technical expertise and needs to be performed in well-equipped setups ${ }^{6}$. This research intends to compare the single-stage ERCP with its two-stage variant, hoping to generate evidence that shall offer greater insight regarding the better technique offering maximum patient benefit.

\section{Methodology}

This prospective analysis was conducted on a sample of 190 diagnosed cholelithiasis patients with choledocholithiasis from May 2015 and November 20I9, scheduled to undergo surgery at the Ziauddin University Hospital, Karachi. The sample was collected using non-probability, consecutive sampling. After taking written informed consent, the basic sociodemographic data and details such as operative time, hospital stay, success, or failure of common bile duct clearance and postoperative complications were recorded onto a pre-structured questionnaire and analyzed using SPSS Version 23.0 \& Microsoft Excel 2016.

All consenting pre-diagnosed patients of cholelithiasis with choledocholithiasis as per the American Society of Anesthesiologists (ASA) classification-ASA Class I, II and III, of either gender and aged between 20 to 70 years were included. Patients presented with comorbid conditions such as bleeding disorders or coagulopathies, carcinoma of the head of the pancreas, peri-ampullary carcinoma, acute pancreatitis or acute cholangitis and major systemic diseases such as diabetes mellitus were excluded from the study. Also excluded were the patients with ASA class IV \& V and redo cases.

The included patients were then divided into two groups, Group A patients underwent ERCP along with LC in a single setting under general anesthesia. The patients first underwent ERCP in prone position followed by standard 4 port LC in the supine position. The protocol in place was that in case of failure 
of ERCP to clear the stones, laparoscopic $\mathrm{CBD}$ exploration shall be attempted and if that also failed, the procedure will be converted to open CBD exploration followed by open cholecystectomy and placement of T-tube. Patients in Group B first underwent ERCP with sphincterotomy and stone extraction from common bile duct by the gastroenterologist as an outdoor procedure under total intravenous anesthesia. Patients were observed for 3 hours post-procedure for any immediate complications and then discharged. Group B patients were again called for an interval LC after a period determined by the general surgeon, (maximum I4 days as soon as the inflammation subsided sufficiently and surgical field is cleared) as an elective case in the main operation theatre. Standard 4 port LC technique was employed. The protocol dictated that in cases of failure of ERCP in the two-stage group, a repeat attempt at clearance will be done after one week and if it fails again laparoscopic CBD exploration will be attempted as in single-stage group and if that also fails, the procedure will be converted to open CBD exploration followed by open cholecystectomy and placement of T-tube.

\section{Results}

Among the I90 patients enrolled in the study, I $24(65 \%)$ were females while the remaining $66(35 \%)$ were males. The mean age of the sample stood at $38 \pm 7.1$ years. Both procedural variants yielded similar success rates. No adverse effects were observed among the patients of both groups. The success rate was higher among the patients of group A $(96.79 \%)$ as compared to $90.53 \%$ among group B patients.

Table I: Procedural Comparison $\&$ Success rate among the participants of the two study groups; Single-stage \& Two-stage ERCP

\begin{tabular}{lcc}
\hline Characteristics & Group A (n=95) & Group B (n=95) \\
\hline Success Rate (\%) & 96.79 & 90.53 \\
\hline Mean Operative Time (Mins) & $57 \pm \mathrm{I} 2$ & $4 \mathrm{I} \pm \mathrm{I} 6$ \\
\hline Mean Hospital Stay (Hours) & $\mathrm{II0} \pm 57.6$ & $\mathrm{I} 48 \pm 27.2$ \\
\hline Difficulty Cannulation & $05(5.26)$ & $\mathrm{II}(\mathrm{II} .57)$ \\
\hline Residual CBD Stones & OI $(\mathrm{I} .05)$ & $0 \mathrm{I}(\mathrm{I} .05)$ \\
\hline Post-Operative Pancreatitis & O2(2.10) & $07(7.36)$ \\
\hline Convert to Open Procedure & OI $(\mathrm{I} .05)$ & $0 \mathrm{I}(\mathrm{I} .05)$ \\
${ }^{*}$ Values are given as Mean \pm SD or $\mathrm{n}(\%)$ & & \\
${ }^{*}$ CBD- Common Bile Duct & &
\end{tabular}

\section{Discussion}

Despite the climbing incidence of problems associated with gall bladder, there is a paucity of data regarding the management of common bile duct stones in Pakistan with no previous studies comparing single-stage ERCP \& LC with two-stage ERCP followed by interval LC. International literature comparing similar techniques have yielded variable results ${ }^{7-9}$.

The available evidence suggests that singlestage ERCP \& LC is probably a safer and effective treatment modality but needs validation with research in our local setups. The results of this research may help establish better evidence-based practices regarding the management of choledocholithiasis in our population ${ }^{8}$. 
The results were in line with our hypothesis, Group A yielded overall better outcomes than group B. Additionally, the prevalent belief that single-stage surgery is too difficult was refuted by our results which showed that no additional difficulty was reported by operating surgeons during cannulation. A study by Selimah et al., in 2016 reported that the ERCP cannulation rate was $97.5 \%$ in both groups. Complete CBD clearance was accomplished in $82.5 \%$ of patients in the two-stage group versus $80 \%$ of patients in the single-stage group $(\mathrm{p}>0.05)$. This is synonymous with the findings of our study which yield similar CBD clearance rates in both group?.

Morino et al., from in Italy in 2006 reported that complete CBD clearance was accomplished in $80 \%$ patients in the two-stage group versus $95.6 \%$ patients in a single-stage group $(p=0.06)$. Our research also has a similarly high clearance rate. Postoperative pancreatitis occurred in $2.2 \%$ of patients versus $0 \%$ of patients between the two groups respectively which was not statistically significant. The mean hospital stay between the two groups (4.3 days in single stage versus 8 days in the two-stage group) was found to be statistically significant $(p<0.000 I)^{10}$. The results of our study were just as encouraging.

Sahoo et al., from India in 2014 reported that the ERCP cannulation rate was $90.2 \%$ in one stage group and $90.5 \%$ in the two-stage group $(p>0.05)$. Though our results showed the two procedures to be slightly less similar in terms of outcome. Complete CBD clearance was accomplished in $71 \%$ of patients in two-stage group vs $90.5 \%$ patients in a single-stage group $(p<0.05)$. In the two-stage group, postERCP serum amylase was raised in $22 \%$ of patients with $12 \%$ of patients having severe pancreatitis versus $0 \%$ of patients with hyperamylasaemia or pancreatitis in one stage group. The mean hospital stay was 6.8 days in a single-stage group vs 10.9 days in two-stage groups respectively $(p<0.05)$. Our mean hospital stay however was much lesser ${ }^{\mathrm{II}}$.

Another study by Ghazal et al., from Egypt in 2009 studied one stage technique and reported that the CBD cannulation was successfully performed in $91.7 \%$ cases with a stone clearance rate of $100 \%$, supporting our claim that no additional difficulty is attributed to this procedure than the conventional procedures $^{12}$. There was no case of postoperative pancreatitis and the mean hospital stay was $2.55 \pm 0.89$ days. The mean operation time was II9.09 \pm I4.4 minutes. Bansal et al., in 2010 found a successful stone clearance rate of $73.3 \%$ in two-stage technique $^{\mathrm{I2}, \mathrm{I3}}$. Hence clearance rates are encouraging in research from other parts of the world as well. A meta-analysis by Tan et al., published in 2017 found that the success rate of CBD clearance was $93 \%$ in a single-stage group versus $92 \%$ in the two-stage group, the difference was statistically insignificant $(\mathrm{p}=0.60)$. The incidence of pancreatitis was found to be lesser $(0.6 \%)$ in the single-stage group versus $(4.4 \%)$ in the two-stage group $(p=0.0 I)^{I 4}$. This trend was mirrored in this research as well, thus cementing this finding as applicable in different settings.

One of the major limitations of our study was the lack of research on patient satisfaction and quality of life. Furthermore, the pain examination could also be used to draw significant inferences in favor of single-stage ERCP \& LC.

\section{Conclusion}

After careful consideration, it can be concluded that patients in group A may yield better 
surgical outcomes giving single-stage ERCP \& LC the edge over two-stage ERCP followed by interval LC. Thus, single-stage ERCP \& LC may be recommended as the method of choice as it is cost-effective, decreases overall hospital stay and there are lesser chances of PostOperative Pancreatitis with single-stage ERCP \& LC as compared to two-stage.

\section{Conflicts of Interest}

None.

\section{Acknowledgment}

We acknowledge the scientific assistance and technical support provided by LUMHS Research Forum.

\section{Funding}

None.

\section{References}

I. Boberg KM. The Clinical Burden of Biliary Disease: A Global Perspective. Springer. 2017;8(I):264-266.

2. Coccolini F, Catena F, Pisano M, Gheza F, Fagiuoli S, Di Saverio S, Leandro G, Montori G, Ceresoli M, Corbella D, Sartelli M. Open versus laparoscopic cholecystectomy in acute cholecystitis. Systematic review and meta-analysis. Int J Surg. 2015;18:196-204.

3. Saia M, Mantoan D, Buja A, Bertoncello C, Baldovin T, Callegaro G, Baldo V. Time trend and variability of open versus laparoscopic cholecystectomy in patients with symptomatic gallstone disease. Surg. Endosc. 2013;27(9):3254-326I.

4. Bilal M, Haseeb A, Saad M, Ahsan M, Raza M, Ahmed A, Shahnawaz W, Ahmed B, Motiani V. The Prevalence and Risk Factors of Gall Stone among Adults in Karachi, South Pakistan: A Population-
Based study. Glob J Health Sci. 2016;9(4):I06-II4.

5. Ghazanfor R, Liaqat N, Changeez $M$, Tariq M, Malik S, Ghazanfar KR, Khan JS. Choledocholithiasis: Treatment Options in a Tertiary Care Setup in Pakistan. Cureus. 2017;9(8):eI58.

6. Fagiuoli S, Di Saverio S, Leandro G, Montori G, Ceresoli M, Corbella D, Sartelli M, Sugrue M, Ansaloni L. Open versus laparoscopic cholecystectomy in acute cholecystitis. Systematic review and meta-analysis. Int J Surg. 20I 5;30:I-9.

7. Costi R, Gnocchi A, Di Mario F, Sarli L. Diagnosis and management of choledocholithiasis in the golden age of imaging, endoscopy and laparoscopy. World J Gastroentero. 20I4; 20(37):I3382.

8. Singh AN, Kilambi R. Single-stage laparoscopic common bile duct exploration and cholecystectomy versus two-stage endoscopic stone extraction followed by laparoscopic cholecystectomy for patients with gallbladder stones with common bile duct stones: systematic review and metaanalysis of randomized trials with trial sequential analysis. Surg. Endosc. 2018;32(9):3763-3776

9. Selimah MA, El Sherief AM, Anwar MM. Comparison of one-stage endoscopic retrograde cholangiopancreatography and cholecystectomy versus two-stage endoscopic retrograde cholangiopancreatography and cholecystectomy for treatment of cholelithiasis with choledocholithiasis. Egypt J Surg. 2016;35(4):398-402.

I0. Morino M, Baracchi F, Miglietta C, Furlan N, Ragona R, Garbarini A. Preoperative endoscopic sphincterotomy versus laparoendoscopic rendezvous in 
patients with gallbladder and bile duct stones. Ann. Surg. 2006;244(6):889896.

II. Sahoo MR, Kumar AT, Patnaik A. Randomised study on single stage laparoendoscopic rendezvous (intra-operative ERCP) procedure versus two stage approach (Pre-operative ERCP followed by laparoscopic cholecystectomy) for the management of cholelithiasis with choledocholithiasis. J Minim Access Surg. 20I4;IO(3):I39-I43.

I2. Ghazal AH, Sorour MA, El-Riwini M, El-Bahrawy H. Single-step treatment of gall bladder and bile duct stones: A combined endoscopic-laparoscopic technique. Int J Surg. 2009;7(4):338346.

I3. Bansal VK, Misra MC, Garg P, Prabhu M. A prospective randomized trial comparing two-stage versus single-stage management of patients with gallstone disease and common bile duct stones. Surg. Endosc. 2010;24(8):1986-1989.

I4. Tan C, Ocampo O, Ong R, Tan KS. Comparison of one stage laparoscopic cholecystectomy combined with intraoperative endoscopic sphincterotomy versus two-stage pre-operative endoscopic sphincterotomy followed by laparoscopic cholecystectomy for the management of pre-operatively diagnosed patients with common bile duct stones: a meta-analysis. Surg. Endosc. 2018;32(2):770-778. 